\title{
Allocentric location cues and the visual guidance of walking towards seen targets
}

\author{
Tracey A. Herlihey", Danlu Cen, Seralynne D. Vann, Cyril Charron \\ \& Simon K. Rushton\#
}

School of Psychology, Cardiff University, Tower Building, Park Place, Cardiff, CF10 3AT, UK

\section{\# Correspondence}

\author{
Simon Rushton \\ School of Psychology \\ Cardiff University \\ Park Place \\ Cardiff, CF10 3AT \\ Wales, UK \\ rushtonsk@cardiff.ac.uk \\ +442920870086
}

Running head: Allocentric location cues and walking

Declarations of interest: none

${ }^{*}$ Current address: Healthcare Safety Investigation Branch, Fox Lane, Farnborough, Hampshire, United Kingdom. 


\section{Abstract}

2 We hypothesised allocentric location cues (information about the location of an

3 observer relative to the surrounding environment) have a role in the on-line visual

4 guidance of walking towards seen target objects. We used a standard technique for

5 assessing the contribution of cues when walking to targets: we perturbed the

6 perceived egocentric direction of the target, and measured the curvature of the

7 resultant walking trajectories. By removing motion cues we were able to isolate

8 allocentric location cues and quantify their utility. Enclosed, geometrically regular,

9 environments contain rich allocentric location cues. In the first experiment

10 participants walked through a large enclosed outdoor space; allocentric cues were

11 isolated by synchronised eye closing and stepping. In the second experiment

12 participants walked through a smaller enclosed indoor space; allocentric cues were

13 isolated with stroboscopic lighting. Both experiments provided support for the

14 hypothesis that, when they are available, allocentric location cues have a key role

15 when walking to seen targets.

16

17 Keywords: Walking; optic flow; allocentric location; egocentric direction; visually

18 guided action; perception and action 


\subsection{Introduction}

An extensive body of neurophysiological work has shown that a rodent's location relative to its surrounding environment, its world-relative location, or allocentric location, is coded in spatially-responsive neurons in the hippocampal formation and surrounding neural areas (Moser, Kropff \& Moser, 2008) (O’Keefe and Dostrovsky, 1971). Evidence suggests a comparable neural representation of allocentric location in humans (Doeller, Barry, \& Burgess, 2010; Ekstrom et al., 2003; Jacobs et al., 2013; Miller et al., 2013) and it has been reported that scene-selective neural areas code the distance and orientation of large surfaces (Lescroart \& Gallant, 2019).

Here we examine the role of allocentric location cues in the online visual guidance of walking to a seen target. To date, research has focussed on two sets of cues: visual motion cues, and egocentric direction cues. The most well-known motion cue is optic flow (Gibson, 1954/2009, 1958/1994). When an observer moves forward a radial pattern of optical motion is created at the eye (Figure 1a). The centre of the radial pattern indicates the direction of translation (the "heading" direction). Other prominent motion cues include differential motion parallax (Cutting et al, 1992) and target drift (the motion of the target relative to the observer, or a point fixed relative to the observer; Llewellyn, 1971). Egocentric direction (Rushton, Harris, Lloyd and Wann, 1998; Figure 1b) is the direction of the target relative to the observer's body: if the observer regulates locomotion direction to keep the target at a fixed direction, typically straight ahead, he or she will reach the target.

We hypothesised that allocentric location cues play a role in not just navigation to an unseen target and path integration (e.g. Bush, Barry, Manson \& Burgess, 2015), but also in the visual guidance of walking to seen targets. 

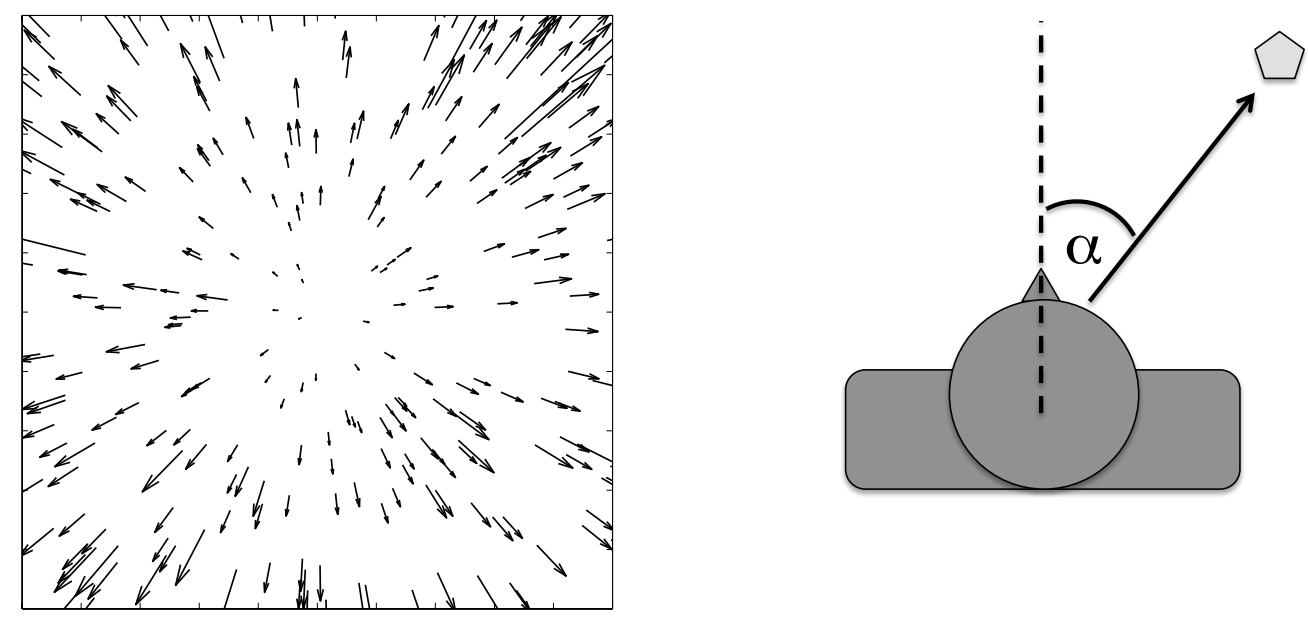

Figure 1. . Radial optic flow field, the pattern of motion available at the eye of an observer moving forward. An observer would reach a target by keeping the centre

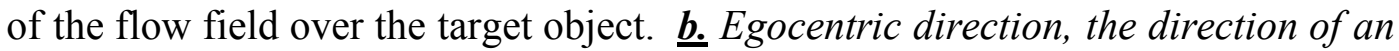
object relative to the body. An observer would reach a target by walking so as to keep the target perceptually straight ahead, i.e. at $\alpha=0$.

The use of allocentric location cues in the visual guidance of walking is hinted at in existing studies. In the published research there has been disagreement about the contribution of optic flow and egocentric direction cues. A common feature of studies that report evidence for use of optic flow, is the use of geometrically regular enclosed environments (e.g., Warren et al., 2001; Turano, Yu, Hao, \& Hicks, 2004).

51 A common feature of those that do not report evidence for use of optic flow is the use 52 of open and irregularly shaped environments (e.g., Rushton, et al, 1998; Saunders, \& 53 Durgin, 2011). The more plentiful allocentric location cues in the enclosed 54 environments could explain the difference. 

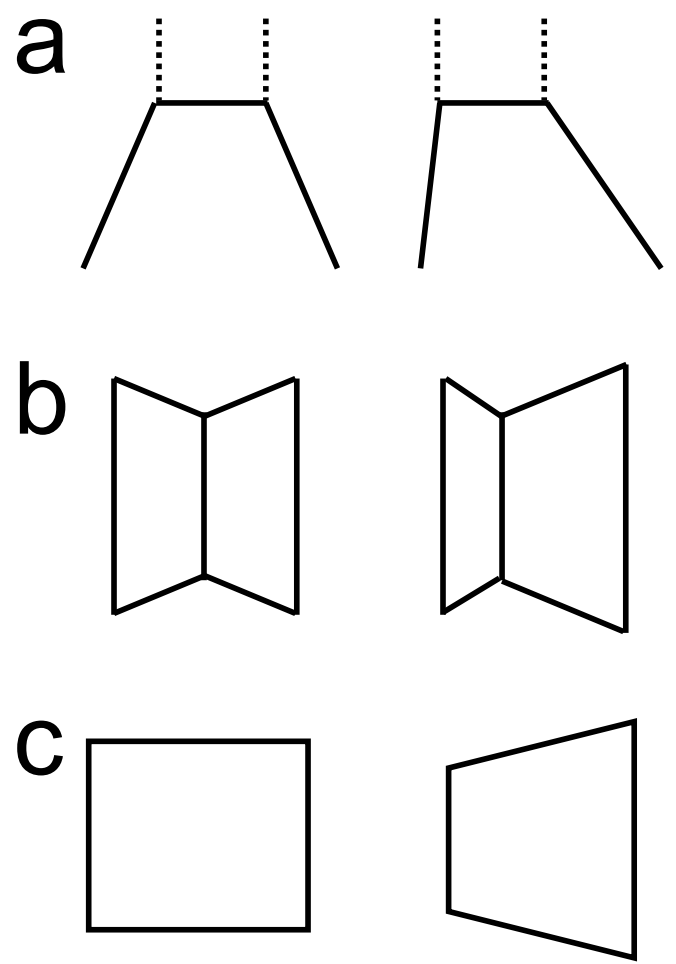

Figure 2: Perspective cues in the retinal images that provide information about allocentric location. a. The angle formed by the bottom of two side walls and the far wall (see splay angle; Beall and Loomis, 1996), is informative about lateral position. In the left image the observer is located halfway between the walls, in the right the observer is located towards the left. b. The perspective shape of an interior corner is informative about position relative to the corner (Beusmans, 1997). In the left image the observer is located directly ahead of the corner, in the right image the observer is stood to the left. c. Perspective shape of a wall. In left image observer is located at central location facing the wall. In the right image, the observer is stood to the right, facing to the left.

Observers could use a variety of sources of visual information to infer allocentric location, such as the distances to surrounding walls. A particularly rich source of

59 information on allocentric location comes from perspective cues. For example, lateral position in a room or corridor, is indicated by the visual angles of the lines formed by 
61 the bottom edges of the left and right walls in the retinal image (splay angle; Beall and Loomis, 1996; Figure 2a). The relative image size of the left and right walls (or texture or features on the walls) also provides information about lateral position. Position relative to a corner is indicated by the perspective shape of the image of the wall (Figure 2b; Beusmans, 1997). Orientation relative to a wall ahead is indicated by the perspective shape of the wall (Figure 2c) in the retinal image.

We have highlighted perspective cues here for illustrative purposes but other cues could provide information about allocentric location. For example, absolute and relative distance cues (see Cutting \& Vishton, 1995 for an overview of cues to distance) provide information about the position of the observer relative to the

71 surrounding environment.

To reach a seen target using allocentric location cues, the observer would monitor and regulate their location relative to the surrounding environment, using allocentric location cues - rather than monitoring motion, or egocentric direction cues.

We designed an experiment to test our hypothesis that allocentric location cues provide important information for the visual guidance of walking to a seen target. We used prism glasses (a standard tool that rotates the visual scene to the left/right relative to the observers' head) to selectively perturb the perceived egocentric

79 direction of a target $\sim 17 \mathrm{~m}$ away (see Figure 3 ). If no cues other than egocentric direction are used the observer would take a predictable curved trajectory to the target

81 (Rushton et al, 1998). If the trajectory is straighter than the "egocentric trajectory"

82 then this indicates other cues are playing a role. The straightness of the trajectory

83 indicates the strength, or contribution, of the other cues. 
(a)

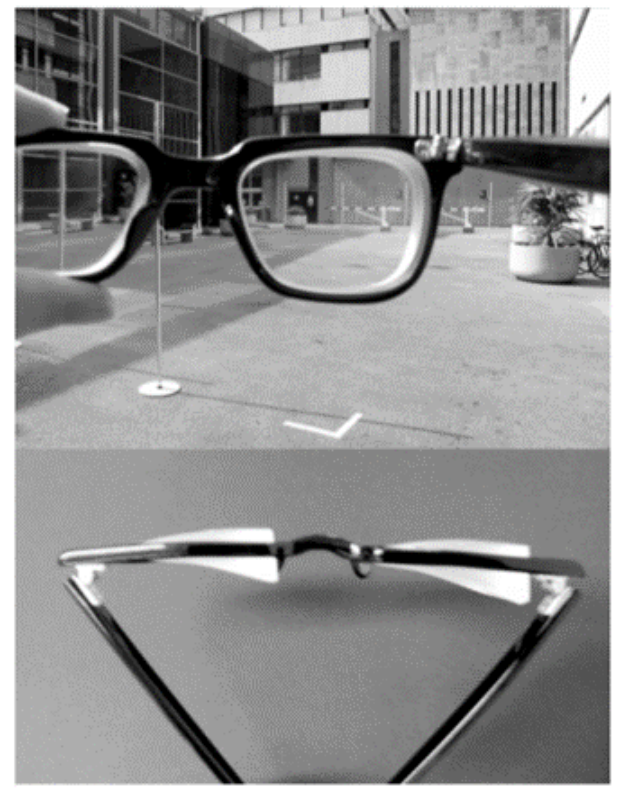

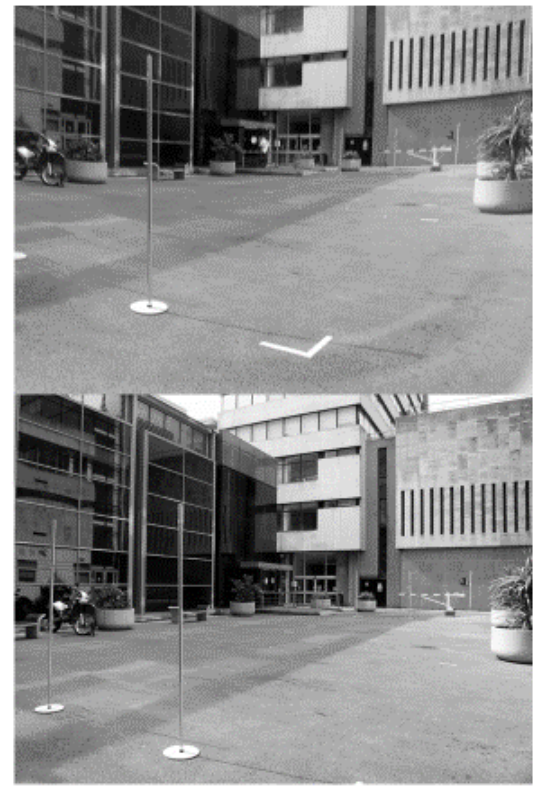

(c)

(d)

Figure 3: (a) and (b) Base right, leftward displacing prisms. Both (c) and (d) show the experimental area. (c) was taken through the leftward prisms shown in (b). (d) was taken without prisms. The animal literature suggests that the neural coding of location stabilises over time (Frank et al. 2004), therefore we measured walking over an extended period, 48 trials, to see if the trajectories changed. However, to be able to assess if there was a change in the use of allocentric location cues it is necessary to account for changes that occur 
in perception of egocentric direction. In earlier work (Herlihey \& Rushton, 2012) we found that perceived egocentric direction changes after walking with prisms in the same environment. So we could factor out any changes in trajectory due to changes in perceived egocentric direction, and isolate any change due to use of allocentric location cues, we inserted measures of perceived straight-ahead into the experiment. To anticipate the results, in the NoMotion condition we found trajectories were less curved than would be expected if egocentric direction was the sole cue used. This suggests that allocentric location cues provide valuable information for the visual guidance of walking to a seen target. In addition, the presence of motion cues (continuous or discontinuous) had little impact, which raises questions about the role or necessity of motion cues.

Experiment 1 was conducted in an outdoor space. We tested, and supported, our key conclusion about the role of allocentric location cues in a replication, Experiment 2, which was conducted in a large room, using a strobe lighting to isolate allocentric location cues.

\subsection{Experiment 1}

\subsection{Methods}

\subsubsection{Participants}

Sixty-five (11 male) right-handed healthy participants with normal, or corrected to normal (by contact lenses only) vision from Cardiff University took part in return for course credit. Five participants were unable to complete the experiment due to weather conditions (the experiment took place outside). The remaining sixty participants were evenly split across three conditions (see below). The research was 
121 carried out in accordance with the Declaration of Helsinki, all participants gave

122 informed consent and the School of Psychology's Ethics Committee approved the

123 study.

124 As there is no prior research that addresses the question we were interested in we

125 were unable to perform an a priori power analysis, therefore for Experiment 1 we

126 were guided by prior literature. Previous research by Bruggeman \& Warren

127 (Bruggeman, Zosh, and Warren, 2007; Bruggeman and Warren, 2010) used 16 or 20

128 participants, others have used fewer, e.g. Saunders and Durgin (2011) and Rushton et

129 al (1998). We chose to go with the largest group size, 20 participants per condition.

130 We use $95 \%$ CIs to convey the precision of the measurements and Bayes Factors to

131 evaluate the relative weight of evidence for and against differences.

132

133

134

135

\subsubsection{Experimental setup}

Participants walked within an outdoor environment (see Figures 3 and S1). Four buildings enclosed the space. A number of objects, including plant pots, benches and bicycle racks were arranged around the edges of the environment.

To produce a shift in the perceived direction of the target object, participants wore high quality, custom made, binocular prism glasses (see Figure 3) that produced a lateral rotation of $8.5^{\circ}$. For each condition, ten participants wore base left, rightward displacing prisms, and ten wore base right, leftward displacing prisms.

Three target posts were located at one end of a 17 x $5 \mathrm{~m}$ rectangular area (Figure S1), and two were located at the opposite end. Luminous material was attached to the posts a little below eye height $(1.5 \mathrm{~m})$. Participants were required to walk back and forth between the posts as instructed by the experimenter while maintaining fixation on the luminous material (see Procedure). 


\subsubsection{Experimental conditions}

The three conditions (Motion, StopGo, NoMotion) were described briefly above. In the Motion condition participants walked at a natural pace to the target. This condition allowed us to assess how people walk normally in a typical full cue environment and it served as a reference for comparison. The StopGo condition required the participants to bring both feet together after each step, and pause momentarily, before proceeding. This condition allowed us to determine the effect of discontinuous stepping, and hence optic flow. In the NoMotion condition, participants performed the same stepping actions as in the StopGo condition but they only opened their eyes while they were stationary (i.e., eyes were closed while stepping towards the target and only open when pausing between steps). This condition was the one of primary theoretical interest as it removed optic flow while preserving the allocentric location cues. Further information on the visual information available in the StopGo condition is provided in the supplemental materials [see HerliheySM.pdf].

\subsubsection{Procedure}

At the beginning of the trial the participant closed his or her eyes before donning the prism glasses. Next, with eyes closed, the participant was rotated 1.5 times so that he or she was facing in the general direction of the target (located at the other end of the rectangular area, at a distance of approximately $17 \mathrm{~m}$ ). This routine was used to (i) prevent participants from seeing a shift in visual direction that would have been apparent if they put on the prism glasses while stood still with eyes open, and (ii) prevent participants from correcting for the displacement of the visual image with a change in head orientation. The experimenter informed the participant of their target, then, with eyes open, the participant turned and faced the target before the trial began. 
Each participant walked 48 trajectories in total, taking on average 40 minutes to complete the experiment, walking a total of approximately 770 metres.

Measures of perceived straight-ahead (see below) were taken without prisms prior to initial exposure, and after four phases: phase 1, after 6 trajectories: phase 2, after a further 6 trajectories (total 12): phase 3, after a further 12 trajectories (total 24): phase 4, after a further 24 trajectories (48 in total). After each phase, the participant was asked to close his or her eyes and was guided to the 'measurement area' located approximately $5 \mathrm{~m}$ away from the walking area (Figure S1); measurements of straight ahead were then re-taken. After the measurements, with eyes closed, the participant was guided back to the 'walking area'.

\subsubsection{Measurement of walking trajectories}

Head and target position were recorded in a plan view using a Sony Ex Wave HAD

colour video camera (running at $50 \mathrm{~Hz}$ ), mounted on the twelfth-floor balcony of one of the surrounding buildings. The videos were processed using custom MATLAB image processing routines. Walking direction (target-heading angle) was calculated by taking the simultaneous angle between the target position and the participant's instantaneous direction of locomotion (tangent to the curve) at each point as they travelled through the environment. Since the direction of heading error differed according to the direction of the prism, we flipped the sign on the right prism data so that it could be combined with the left prism data. In line with standard practice, (e.g., Warren et al., 2001) we removed the first metre of the beginning and end of the trajectories from the analysis due to noise. We also excluded any data points that were more than 3 SDs from the mean (defined over observers for the given distance and given trial) or more than $10^{\circ}$ from the expected range of $0-8.5^{\circ}$. 


\subsubsection{Measurement of perceived direction}

The literature on the coding of allocentric location in rodents indicates that over time, experience of, and attention to, the environment leads to the development of a stable representation of the scene (Frank et al., 2004; Kentros et al. 2004; Monaco et al. 2014). It would be of significant interest if we could find evidence of the development of a representation of allocentric space. Such a development might be revealed by a change in walking trajectory: as the representation became richer, so its contribution to the visual guidance of walking increases and the trajectories straighten. Based on previous work (e.g. Bruggeman \& Warren, 2007) we anticipated that trajectories would straighten over trials in our experiment. The problem is that straightening could be due to a different factor, it could result from changes in perceptuo-motor alignment: discrepant optic flow has been shown to shift perceived egocentric direction (via a shift in perceived visual straight-ahead; see Held \& Freedman, 1963; Held \& Mikaelian,1964; .Herlihey \& Rushton, 2012); a change in perceived direction would lead to a change in the curvature of the walking paths (see Rushton \& Salvucci, 2001). To address this issue we interleaved measures to track changes in perceptuo-motor alignment. We used two standard measures: Visual straight-ahead (VSA) is a measure of alignment of the eye-head system and proprioceptive straight-ahead (PSA), a measure of alignment in the limb-head system (see Redding \& Wallace, 1997, for an overview of measures of perceived direction).

Participants were stood two metres from a wall. Attached to the wall was a ruler with small numbers only visible to the experimenter. We measured VSA by asking participants to verbally indicate when a visual target (a light spot moved by the experimenter along the top of the ruler) was straight-ahead. We measured PSA by asking participants to close their eyes, and position their extended right arm so that it 
221 was pointing straight-ahead. When the participant was confident he or she was 222 pointing to straight-ahead, the participant turned on a laser pointer held in their

223 pointing hand; the experimenter recorded the position of the light spot. The position

224 of the participant was changed at each measurement phase to prevent use of a

225 remembered point on the wall. We measured VSA and PSA five times during the

226 experiment: prior to walking, and after trajectories 6, 12, 24 and 48 (the final

227 trajectory). We combined data for left and rightward prisms by flipping the sign on

228 the right prism data. Changes in perceived direction (VSA and PSA) were calculated

229 by subtracting pre-exposure measures.

\subsection{Results}

232 Figure 4a shows the mean target-heading angle over trajectories for each condition.

233 To aid visual comparison, the three conditions (NoMotion, StopGo and Motion) are

234 overlaid and error bars are shown consecutively over conditions, on every third data 235 point. To recap, a target-heading angle less than $8.5^{\circ}$ indicates the use of cues other 236 than egocentric direction. In panel B we show the difference between the conditions 237 expressed as t-values. 

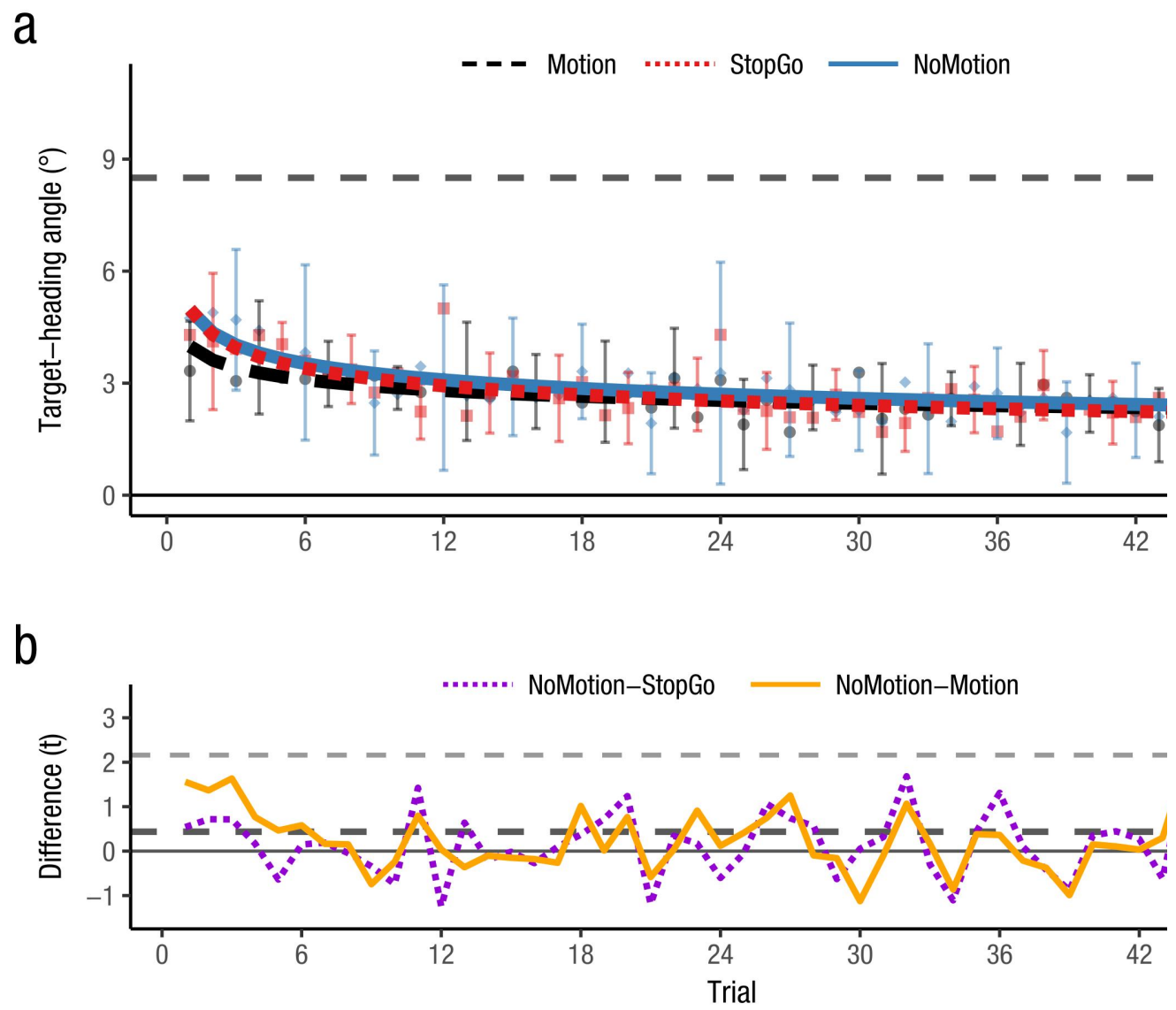

Figure 4. a. Mean target-heading angle across all trajectories. Mean over trial $(\sim 16.5$ m) and observers ( $\mathrm{n}=20$ per condition) target-heading angle is shown on the vertical axis. Dashed grey line at $8.5^{\circ}$ indicates optical displacement of the prisms. Error bars represent 95\% confidence intervals. Solid blue line is NoMotion condition; dashed black line is Motion condition; dotted red line is StopGo condition. Data fit with best-fit (logarithmic) curves. $\boldsymbol{b}$. Differences (t-values), in target-heading angle as a function of trial number. NoMotion-Motion solid line (orange), NoMotion-StopGo dotted line (purple). The dark grey dashed line indicates the $\mathrm{t}$-value for $\mathrm{BF}_{10}=1 / 3$. Points below the line indicate "substantial" (Kass \& Raftery, 1995) evidence the target-heading angle in the NoMotion is not larger than the comparison condition. The light grey dashed line indicates the $\mathrm{t}$-value for $\mathrm{BF}_{10}=3$. Points above the line indicate substantial evidence that the target-heading angle in the NoMotion is significantly larger than the other condition. Test are 1-tailed with predicted order Motion $<$ StopGo $<$ NoMotion. 
239 Visual inspection of Figure 4a reveals that in the key NoMotion condition, target-

240 heading angle was considerably less than the displacement of the prism on every trial

241 (t-tests, all $\mathrm{BF}_{10}>20$; Cohen's $\left.\mathrm{d}>0.80\right)$. Given there was no optic flow in this

242 condition we can interpret this data as providing strong evidence that allocentric

243 location cues can play a key role in the visual guidance of walking.

245 Next we examined what happens when flow cues are added by comparing the target-

246 heading angle in the NoMotion condition to the Motion (full flow) and StopGo

247 (intermittent flow) conditions. The mean (over trial) target-heading angles are $2.68^{\circ}$

$248 \quad\left(\right.$ Motion, $\left.\mathrm{SD}=1.21^{\circ}\right), 2.76^{\circ}\left(\right.$ StopGo, $\left.\mathrm{SD}=1.34^{\circ}\right), 2.92^{\circ}\left(\right.$ NoMotion, $\left.\mathrm{SD}=2.14^{\circ}\right) . \mathrm{A}$

249 1-way, 3-level, Bayesian ANOVA provides "substantial” (Kass \& Raftery, 1995)

250 evidence for no difference between the conditions $\left(\mathrm{BF}_{10}=0.15\right.$; Cohen's $\left.\mathrm{d}=0.063\right)$.

252 Because mean measures may obscure important differences on specific trials, e.g. on

253 the first trials, we looked at the trial by trial differences. Figure $4 \mathrm{~b}$ shows the

254 difference, expressed as t-values, in target-heading angle between conditions. Some

255 difference between the NoMotion condition and the other two conditions can be seen

256 over the first 7-8 trials. This suggests that optic flow may have an initial influence but

257 it makes no appreciable difference after a certain distance has been walked.

259 Previous work (e.g., Bruggeman \& Warren, 2010) using enclosed environments 260 demonstrated that target-heading angle changes not only from trial to trial, but also

261 within trajectories. Changes in target-heading angle have been interpreted as

262 indicating the on-line use of optic flow. In Figure 5 we plot the mean target-heading 263 angle as a function of distance walked from the start position. As before, all three 
264 conditions are overlaid and every third error bar is shown; the lower panel shows the

265 differences between the key NoMotion condition and the two comparison conditions

266 expressed as t-values. In line with previous work, the target-heading angle decreases

267 with distance in all conditions (Figure 5a).

269 Critically, the target-heading angle (Figure 5a) is markedly less than the optical

270 displacement of the prism at all distances in all conditions (all BF $10>10^{5}$; Cohen's d

$271>1.8$ ). Figure $5 \mathrm{~b}$ shows the differences (expressed as a t-statistic) between

272 conditions. Until approximately $13 \mathrm{~m}$ the trajectories are broadly similar in all three

273 conditions. In the last few metres (see Figure 5a) target-heading angle is lower (and

274 hence trajectories are straighter) when optic flow is present. This suggests an

275 influence of optic flow, though the influence is small - the reduction in target-heading

276 angle is only $10-15 \%$ larger when optic flow is present. 

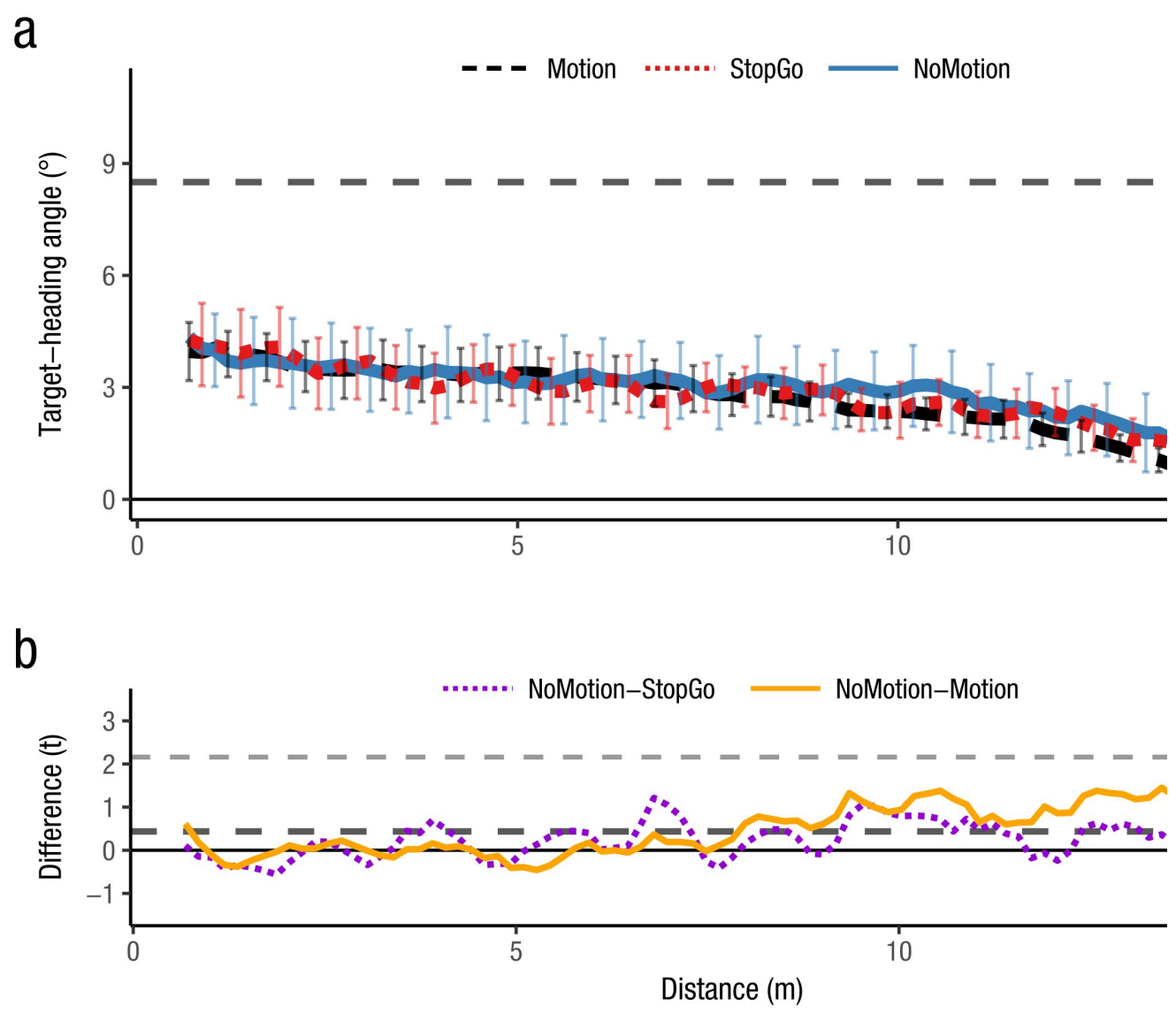

Figure 5. a. Mean target-heading error (averaged over trajectories 1-48) between the first metre and the last metre of a trajectory. Mean (over trial and observers) targetheading angle is shown on the vertical axis. Error bars represent $95 \%$ confidence intervals. Dashed line at $8.5^{\circ}$ indicates optical displacement of the prisms. Solid blue line is NoMotion condition; dashed black line is Motion condition; dotted red line is StopGo condition. b. Differences, as a function of distance plotted as a $t$-value, in target-heading angle. NoMotion-Motion solid line (orange), NoMotion-StopGo dotted line (purple). The dark grey dashed line indicates the $\mathrm{t}$-value for $\mathrm{BF}_{10}=1 / 3$. Points below the line indicate substantial/positive evidence the target-heading angle in the NoMotion is not larger than the comparison condition. The light grey dashed line indicates the $t$-value for $\mathrm{BF}_{10}=3$. Points above the line indicate substantial/positive evidence that the target-heading angle in the NoMotion is significantly larger than the other condition. Test are 1-tailed with predicted order Motion $<$ StopGo $<$ NoMotion. 


\subsubsection{Changes in perceived direction}

279 To recap, we took measures of perceived visual (VSA) and proprioceptive straight-

280 ahead (PSA) so we could factor out their effect on the anticipated (and observed)

281 straightening of trajectories. Figure 6 shows the changes in VSA and PSA. The

282 pattern of PSA changes (Figure 6b) is quite different to the pattern of trajectories

283 (target-heading angle) changes: the PSA changes do not plateau and the order of

284 conditions does not match. The pattern of VSA changes is more similar: VSA

285 (Figure 6a) changed in all conditions, as did the target-heading angle. Changes in

286 VSA also occurred early, similar to the target-heading angle. The only difference is

287 that at the time the first VSA change is measured, the change in the StopGo condition

288 was more similar to the Motion condition than the NoMotion. Given the limited
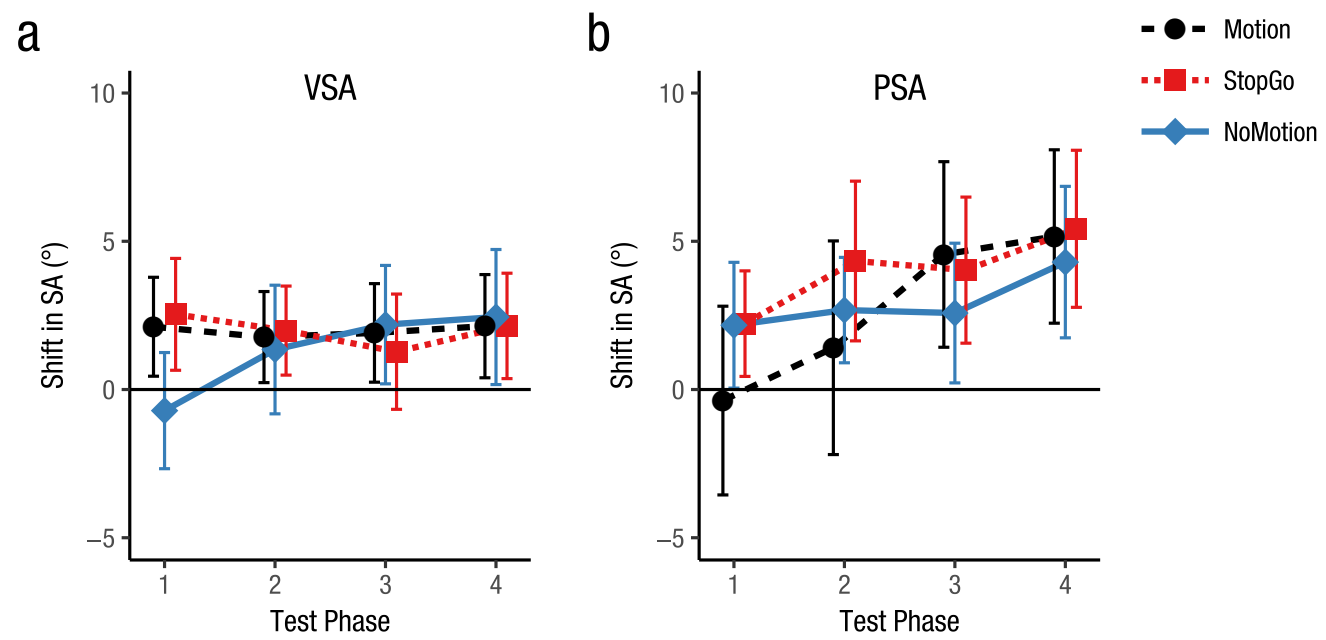

Figure 6. Mean change in VSA (a) and PSA (b) over time. Phase number (corresponding to trajectories $6,12,24$ and 48 respectively) is indicated on the horizontal axis, magnitude of realignment is indicated on the vertical axis. Data from the three conditions are overlaid to aid interpretation. Blue diamond markers are NoMotion, black circular markers are Motion condition, red square markers are StopGo. Error bars indicate 95\% confidence intervals. Herlihey \& Rushton (2010) looked at changes in VSA and PSA after 6 trials. The pattern of VSA and PSA changes over the first 6 trails here is comparable. 
number of data points it is not practical to attempt a formal statistical analysis of the similarity between the changes in VSA and the changes over trajectories. However, given the similarities between the trajectory and VSA changes over the 48 trials, it unwise to interpret the changes in trajectory over trials as having resulted from the development and use of an allocentric representation. It is more parsimonious to assume the changes in trajectory result from a realignment of perceptuo-motor space (specifically perceived VSA).

\section{Experiment 2}

297 The key conclusion of Experiment 1 was that allocentric cues provide an important 298 source of information for the online visual guidance of walking to a seen target. To 299 test the robustness of this conclusion we tested whether the results from the key 300 "NoMotion" condition would replicate in a different environment (an interior space 301 with a closer target), where view of the scene was controlled by strobe flashes rather

302 than by the participant, and when the participants were performing a task that 303 precluded them from attending to their walking.

\section{3.1. Methods}

\subsubsection{Participants}

306 Twelve participants (1 male) took part in the experiment for course credit. All

307 participants had normal or corrected-to-normal vision using contact lenses. The 308 research was carried out in accordance with the Declaration of Helsinki, the study was 309 approved by the School of Psychology Ethics Committee, and informed consent was

310 obtained. The data from Experiment 1, indicated we had sufficient power to justify a

311 large reduction in the number of participants. We decided to reduce to twelve, a

312 somewhat conservative reduction. 


\subsubsection{Experimental setup}

314 The experimental setup was similar to Experiment 1 but for the following differences:

- The environment was a square room $(8.3 \mathrm{~m} \times 8.3 \mathrm{~m} \times 3.4 \mathrm{~m}$, as such

The advantage of using a strobe is that we can guarantee that no motion cues are present because the flash is so brief. The disadvantage is the briefness of the flash impairs the pickup of allocentric location, it is hard to clearly perceive the sides of the room. To minimise the degradation of allocentric cues we used multiple synchronized strobes to maximise brightness and visibility of the room.

\subsubsection{Procedure}

333 The procedure was matched to the NoMotion condition of Experiment 1 but with a 334 few procedural differences. Participants first put on a helmet with trackers and were 335 led to the middle of the room where the trackers were calibrated. After calibration, 336 participants were led, unsighted, back to the starting point. When the participant was

337 ready, the experimenter initiated the trial, and the strobe lights were switched on

338 (flashing for $<10 \mathrm{~ms}$ at $1 \mathrm{~Hz}$ ). To ensure participants did not pay undue attention to 
339 the act of walking, they were required to press a button on a remote controller held in

340 their hand every time the red LED flashed. When they reached half a metre from the

341 target post, a beep sound indicated they should stop. The strobe lights, LED lights

342 and notification beep were controlled by a custom program written using Vizard

343 (version 5, WorldViz, Santa Barbara, USA). Each participant walked 5 trials wearing

344 prism glasses. Given that the majority of the changes in trajectory occurred on the

345 earlier trials of Experiment 1, five trials was judged sufficient to reveal any changes.

346 3.1.4. Measurement of walking trajectories

347 Head position and orientation were tracked and recorded at $60 \mathrm{~Hz}$ using a Vizard

348 program. Walking direction was calculated in the same way as in Experiment 1 and

349 the same rules for removal of outliers were applied.

$350 \quad 3.2$ Results

351 Figure 7 shows the mean target-heading angle for each trajectory. As in Experiment

352 1, the target-heading angle was considerably less than the displacement of the prism

$353\left(8.5^{\circ}\right)$ on every trial (one-sample t-test, all Cohen's $\mathrm{d}>1.11$; all $\left.\mathrm{BF}_{10}>10\right)($ see

354 Figure 7a). The target-heading angle was also considerably less (one-sample t-test,

355 all Cohen's $\mathrm{d}>0.64 ; \mathrm{BF}_{10}>3$ from $0.6 \mathrm{~m}$ ) than the displacement of the prism from the 356 beginning to the end of the trial (Figure $7 b$ ). 
a

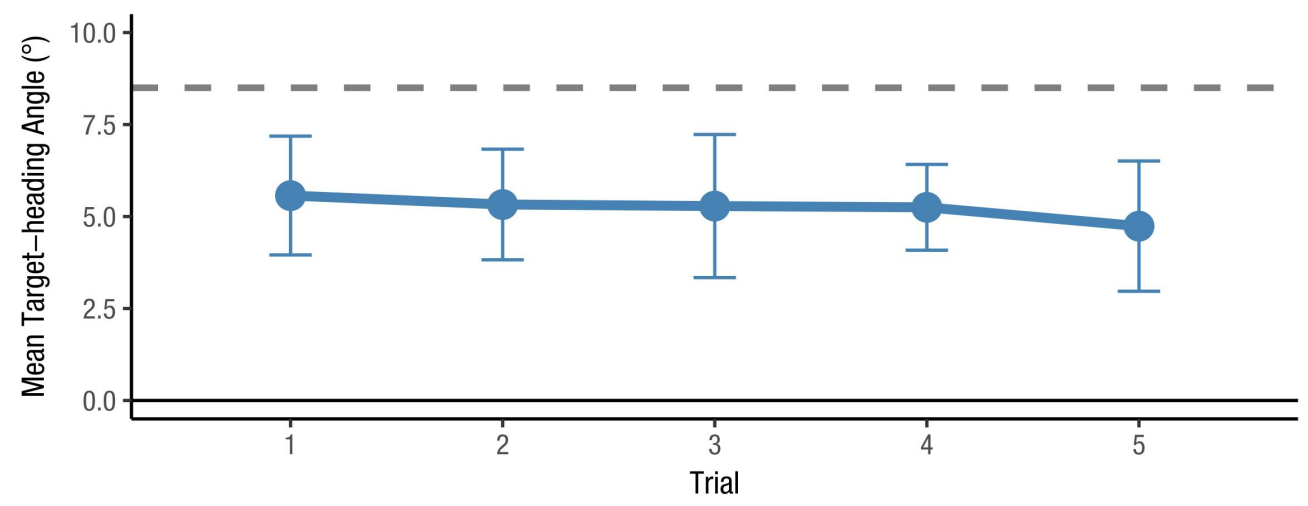

b

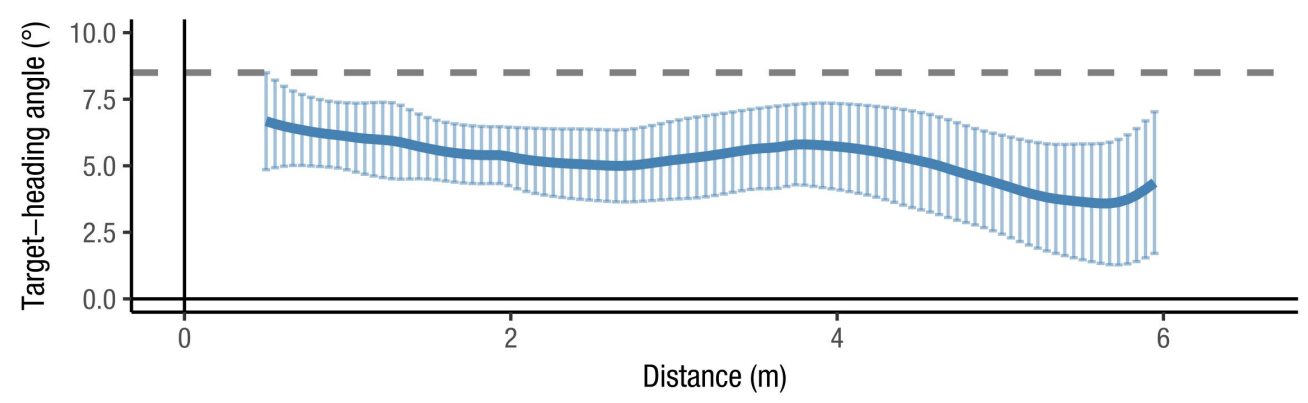

Figure 7. a. Mean target-heading angle across all trajectories. Mean over trial ( $6.5 \mathrm{~m})$ and observers $(n=12)$ target-heading angle is shown on the vertical axis. Dashed line at $8.5^{\circ}$ indicates optical displacement of the prisms. Error bars represent 95\% confidence intervals. b. Mean target-heading error (averaged over trajectories 1-5) between the first half metre and the last metre of a trajectory. Mean (over trial and observers) target-heading angle is shown on the vertical axis. The error bars represent $95 \%$ confidence intervals. Dashed line at $8.5^{\circ}$ indicates optical displacement of the prisms.

357 Despite the differences in environment (room vs. outdoors; $7 \mathrm{~m}$ vs. $17 \mathrm{~m}$ ), access to 358 optic flow information (controlled by the experimenter vs. controlled by the 359 participant), and attentional demands (additional of monitoring task), the pattern of 360 results was similar to Experiment 1. 


\section{Discussion}

The results of Experiment 1 support the hypothesis that when they are salient, allocentric location cues have a role in the online visual guidance of walking to a seen target: the target-heading angle was significantly less than the optical deflection of the prisms $\left(8.5^{\circ}\right)$ in the condition that did not contain optic flow cues (NoMotion). The result of Experiment 2, designed to test this conclusion, using a strobe-illuminated room, provides further support.

\section{Trajectories changed - straightened - over trials. However, the current experiments} provide no evidence that the participants, unlike rodents (Frank et al, 2004), developed a stable internal representation of allocentric space from accumulating experience of the environment. It is possible that a build up did occur, but if so we can conclude it must have occurred rapidly, at a rate too fast for us to detect. The most parsimonious explanation for the changes we observed is that the discrepant optic flow realigned perceived visual straight-ahead (see Herlihey \& Rushton, 2012).

\section{The trajectories we measured in the Motion condition are comparable to those} reported previously by Warren and colleagues, and others (e.g., Warren et al., 2001; Turano et al., 2004). We used a similar enclosed environment (but dissimilar technology) and obtained similar paths. The trajectories are dissimilar to those reported by others (e.g. Rushton et al., 1998; Saunders and Durgin, 2011) who used open environments. This pattern of results across studies is consistent with the conclusion that in open environments egocentric direction is the primary, and often sole, cue, but in enclosed environments, although egocentric cues retain their central role, allocentric location cues have an important part to play. 
388 It would be premature to suggest that there is no influence of optic flow. In the early

389 trials of Experiment 1, there was a consistent (if not statistically significant)

390 difference between the NoMotion condition and the two conditions that contained

391 optic flow. However, it does not follow that any differences reflect the use of optic

392 flow in the online guidance of walking, they could be due to discrepant optic flow

393 biasing perceived visual straight-ahead (VSA) observed over the same period (see

394 Rushton \& Salvucci, 2001).

\subsection{What allocentric location cues are involved?}

397 In the Introduction, we highlighted allocentric location cues that are based on the 398 perspective projection of the environment boundaries (figure 2). We gave distance 399 cues as an example of other cues that provide information about allocentric location.

400 The literature points to some others. For example, Vishton and Cutting (1995), and

401 later, Hahn, Anderson and Saidpour (2003), found observers were able to judge

402 heading direction from snapshots of an environment presented at intervals of more 403 than two seconds apart when the environment contained distinct objects (e.g., real or 404 wireframe trees). There were few objects in our experiments but the relative 405 movement of those in view could have made a contribution. Macuga, Loomis, Beall, 406 \& Kelly (2006) demonstrated that observers were able to judge heading direction in 407 scenes that lack optic flow but do contain stereoscopically defined information about 408 scene layout. The experiments we report were not designed to determine which 409 allocentric location cues were used, but the findings reported here provide a 410 foundation for future work to tease apart different allocentric location cues and 411 determine their role. 


\section{4.2. Use of other strategies or cues in the current experiment?}

414 Observers can perform "blind walking" -view a scene and then walk blindfolded to a 415 target (Thomson, 1983). If observers used blind-walking technique here, we would 416 expect them to take a straight-line trajectory rather than a curving trajectory that is 417 indicative of the continual online regulation of walking direction. Therefore we can 418 rule out this possibility.

420 When a target is close, the drift (change of visual direction) of the object provides

421 information about whether the observer is heading to the left or right of the target 422 (Lewellyn, 1971). No studies have directly examined the use of target drift in 423 walking. One dataset that may demonstrate the utility of target drift is from Harris 424 and Bonas (2002) who found observers walking down a short narrow familiar 425 corridor to an illuminated target while wearing prism glasses, took the same trajectory 426 in the light and dark. One possible explanation of that result is that observers used 427 target drift. In our experiments we removed not only global motion (optic flow) but 428 also object motion (target drift).

\subsection{Relation to animal literature}

431 The finding that allocentric location cues have a role in the online visual guidance of

432 walking to seen targets highlights a potential link between the different literatures.

433 The findings here suggest that allocentric location cues also have a role in the online 434 guidance of human walking. Neurophysiological work on rodents, and imaging work 435 on humans may provide clues about the neural mechanisms that underpin the 
436 437 literature, we predict a difference in neural coding may be found in animals that are 438 439 440

behaviour we observed. Second, based on our results and the broader human moving through natural open and irregular environments (see also Kupric et al., 2014).

\subsection{Combining cues and modelling locomotion}

How would allocentric location cues be combined with other cues? Warren et al. (2001) proposed a simple control model based on a combination of optic flow heading and egocentric direction cues, and others have been proposed since (e.g., Wilkie \& Wann, 2003; Fajen \& Warren, 2007). These models capture behaviour in enclosed environments and could be adapted to accommodate allocentric location cues. An alternative would be to model the behaviour on the assumption that cues are combined to form an internal model of the environment which guides behaviour (Loomis \& Beall, 2004).

\section{Conclusions}

To date, research on the visual guidance of walking to seen targets has focused exclusively on motion cues and egocentric direction cues. The data we report supports the hypothesis that when they are available, as in typical urban enclosed environments, allocentric location cues provide a powerful additional source of information. 
458

459

460

461

462

463

464

465

466

467

\section{Acknowledgements}

We thank Michael Lewis, Rob Honey, Marc Buehner and Paul Warren for feedback on earlier drafts. We thank Harun Karimpur for comments on the penultimate draft.

\section{Declaration of interest}

None.

\section{Supplementary materials}

The supplementary figures, Fig S1-2 referenced in the paper are to be found at TBA.

We also provide additional background information in HerliheySM.doc.

Data is available at [TBA]. The analysis code was made available to reviewers, and is available on request.

\section{Funding}

TAH was funded by an EPSRC studentship, DC was funded by Cardiff University

Overseas Research Scholarship and School of Psychology studentship, and the work was supported by an ESRC grant (ES/M00001X/1) to SKR. SDV is funded by a Wellcome Trust Senior Research Fellowship (WT090954AIA). 


\section{10. Author contributions}

479 TH and SKR designed experiment 1 , TH collected and analysed the data. DC, SKR

480 and SV designed experiment 2, DC collected and analysed the data. CC wrote the

481 image processing code used in Experiment 1. TH and SKR wrote the original ms, 482 DC, SV and CC contributed to revisions. 


\section{References}

Beall, A. C. and Loomis, J. M. (1996) Visual control of steering without course information. Perception, 25, 481-494

Beusmans, J. M. (1997). Perceived object shape affects the perceived direction of self-movement. Perception, 27(9), 1079-1085.

Bruggeman, H., and Warren, W. H. (2010). The Direction of Walking--but Not Throwing or Kicking--Is Adapted by Optic Flow. Psychological Science, 21(7), $1006-1013$.

Bruggeman, H., Zosh, W., and Warren, W. H. (2007). Optic flow drives human visuolocomotor adaptation. Current Biology, 17(23), 2035-2040.

Bush, D., Barry, C., Manson, D., \& Burgess, N. (2015). Using grid cells for navigation. Neuron, 87(3), 507-520.

Cutting, J. E., \& Vishton, P. M. (1995). Perceiving layout and knowing distances: The integration, relative potency, and contextual use of different information about depth. In Perception of space and motion (pp. 69-117). Academic Press.

Doeller, C. F., Barry, C., \& Burgess, N. (2010). Evidence for grid cells in a human memory network. Nature, 463(7281), 657-661.

Ekstrom, A. D., Kahana, M. J., Caplan, J. B., Fields, T. A., Isham, E. A., Newman, E. L., \& Fried, I. (2003). Cellular networks underlying human spatial navigation. Nature, 425(6954), 184.

Epstein, R. A., Higgins, J. S., \& Thompson-Schill, S. L. (2005). Learning Places from Views: Variation in Scene Processing as a Function of Experience and Navigational Ability. Journal Of Cognitive Neuroscience, 17(1), 73-83. 
Fajen, B. R., and Warren, W. H. (2007). Behavioral dynamics of intercepting a moving target. Experimental Brain Research, 180, 303-319.

Frank LM, Stanley GB, Brown EN 2004. Hippocampal plasticity across multiple days of exposure to novel environments. J Neurosci 24: 7681-7689.

Gibson, J. J. (1954). The visual perception of objective motion and subjective movement. Psychological Review, 61, 304, reprinted in Psychological Review, Special Issue: The Centennial Issue of the Psychological Review (1994).

Gibson, J. (1958). Visually controlled locomotion and visual orientation in animals. British Journal of Psychology. 49, 182-94, reprinted in British Journal of Psychology, Special Issue: Celebrating a Century of Psychological Research (2009).

Guterman, P, Allison, R.S and Rushton, SK. (2007). The visual control of walking: do we go with the (optic) flow? Journal of Vision, 7, 1017a.

Hafting, T., Fyhn, M., Molden, S., Moser, M.-B., and Moser, E. I. (2005). Microstructure of a spatial map in the entorhinal cortex. Nature, 436(7052), 801806.

Hahn, S., Anderson, G.J. and Saidpour, A. (2003). Static Scene Analysis for Perception of Heading. Psychological Science, 14, 543-548.

Harris, J. M., \& Bonas, W. (2002). Optic flow and scene structure do not always contribute to the control of human walking. Vision research, 42, 1619-1626.

Harris, M. G., \& Carré, G. (2001). Is optic flow used to guide walking while wearing a displacing prism? Perception, 30, 811-818.

Held R. and Freedman S. J. (1963). Plasticity in human sensorimotor control. Science, $142,455-462$. 
Held R. and Mikaelian H. (1964). Motor sensory feedback versus need in adaptation to rearrangement. Perceptual and Motor Skills, 18, 685-688.

Herlihey, T. A., and Rushton, S. K. (2012). The role of discrepant retinal motion during walking in the realignment of egocentric space. Journal of Vision, 12(3), $1-11$.

Jacobs, J., Weidemann, C. T., Miller, J. F., Solway, A., Burke, J. F., Wei, X. X., ... \& Kahana, M. J. (2013). Direct recordings of grid-like neuronal activity in human spatial navigation. Nature Neuroscience, 16(9), 1188.

Kass, R.E. \& Raftery, A.E (1995). "Bayes Factors" (PDF). Journal of the American Statistical Association. 90 (430): 791

Kentros, C. G., Agnihotri, N. T., Streater, S., Hawkins, R. D., \& Kandel, E. R. (2004). Increased attention to spatial context increases both place field stability and spatial memory. Neuron, 42(2), 283-295.

Lescroart, M. D., \& Gallant, J. L. (2019). Human scene-selective areas represent 3D configurations of surfaces. Neuron, 101(1), 178-192.

Li, L., \& Niehorster, D.C. (2014). Influence of optic flow on the control of heading and target egocentric direction during steering toward a goal. Journal of Neurophysiology,112(4), 766-777.

Llewellyn, K. R. (1971). Visual guidance of locomotion. Journal of Experimental Psychology, 91(2), 245.

Miller, J. F., Neufang, M., Solway, A., Brandt, A., Trippel, M., Mader, I., ... \& Kahana, M. J. (2013). Neural activity in human hippocampal formation reveals the spatial context of retrieved memories. Science, 342(6162), 1111-1114. 
Monaco, J. D., Rao, G., Roth, E. D., \& Knierim, J. J. (2014). Attentive scanning behavior drives one-trial potentiation of hippocampal place fields. Nature neuroscience, 17(5), 725 .

Mollon, J. (1997). “...on the basis of velocity cues alone”: some perceptual themes. Quarterly Journal of Experimental Psychology, 50A, 859-878.

Moser, E. I., Kropff, E., \& Moser, M. B. (2008). Place cells, grid cells, and the brain's spatial representation system. Annu. Rev. Neurosci., 31, 69-89.

O'Keefe, J., \& Dostrovsky, J. (1971). The hippocampus as a spatial map: preliminary evidence from unit activity in the freely-moving rat. Brain research.

Philbeck, J.W. and O'Leary, S. (2005). Remembered landmarks enhance the precision of path integration. Psicológica, 26, 7-24.

Redding, G. M., and Wallace, B. (1997). Adaptive spatial alignment. Mahwah, NJ: Erlbaum.

Rushton, S. K., Harris, J. M., Lloyd, M. R., and Wann, J. P. (1998). Guidance of locomotion on foot uses perceived target location rather than optic flow. Current Biology, 8(21), 1191-1194.

Rushton, S., and Salvucci, D. (2001). An egocentric account of the visual guidance of locomotion. Trends in cognitive sciences, 5(1), 6-7.

Salvucci, D.D. and Gray, R. (2004). A two-point visual control model of steering. Perception, 33, 1233-1248.

Saunders, J. A., and Durgin, F. H. (2011). Adaptation to conflicting visual and physical heading directions during walking. Journal Of Vision, 11(3), 15-15.

Thomson, J. A. (1983). Is continuous visual monitoring necessary in visually guided locomotion? Journal of Experimental Psychology: Human Perception and Performance, 9, 427. 
Turano, K., Yu, D., Hao, L., and Hicks, J. (2004). Optic-flow and egocentric-direction strategies in walking: Central vs peripheral visual field. Vision Research, 45(2526), 3117-3132.

Vishton, P.M. and Cutting, J.E. (1995). Wayfinding, Displacements, and Mental Maps: Velocity Fields Are Not Typically Used to Determine One's Aimpoint. Journal of Experimental Psychology: Human Perception and Performance, 21, 978-995.

Warren, W. H., Kay, B. A., Zosh, W. D., Duchon, A. P., and Sahuc, S. (2001). Optic flow is used to control human walking. Nature Neuroscience, 4(2), 213-216.

Wilkie, R.M. and Wann, J.P. (2003). Controlling steering and judging heading: retinal flow, visual direction, and extraretinal information. Journal of Experimental Psychology: Human Perception and Performance, 29, 363-378.

Wood, R. M., Harvey, M. A., Young, C. E., Beedie, A., \& Wilson, T. (2000). Weighting to go with the flow? Current Biology, 10(15), R545-R546. 